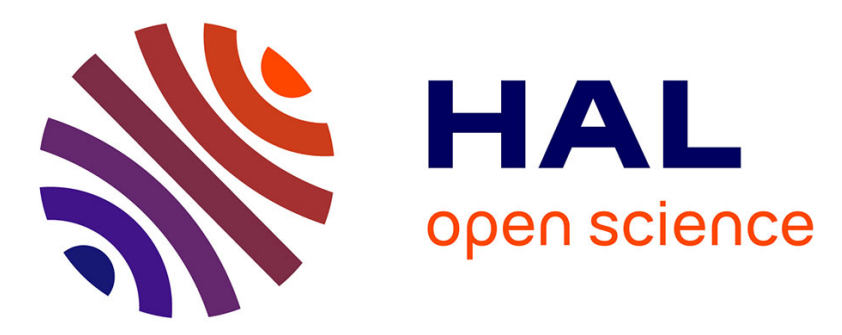

\title{
Towards a Restrained Use of Non-equivocation for Achieving Iterative Approximate Byzantine Consensus
}

Chuanyou Li, Michel Hurfin, Yun Wang, Lei Yu

\section{To cite this version:}

Chuanyou Li, Michel Hurfin, Yun Wang, Lei Yu. Towards a Restrained Use of Non-equivocation for Achieving Iterative Approximate Byzantine Consensus. 30th IEEE International Parallel and Distributed Processing Symposium (IPDPS), May 2016, Chicago, United States. pp.10, 10.1109/IPDPS.2016.62 . hal-01339477

\section{HAL Id: hal-01339477 \\ https://inria.hal.science/hal-01339477}

Submitted on 29 Jun 2016

HAL is a multi-disciplinary open access archive for the deposit and dissemination of scientific research documents, whether they are published or not. The documents may come from teaching and research institutions in France or abroad, or from public or private research centers.
L'archive ouverte pluridisciplinaire HAL, est destinée au dépôt et à la diffusion de documents scientifiques de niveau recherche, publiés ou non, émanant des établissements d'enseignement et de recherche français ou étrangers, des laboratoires publics ou privés. 


\section{Towards a Restrained Use of Non-equivocation for Achieving Iterative Approximate Byzantine Consensus}

\author{
Chuanyou $\mathrm{Li}$ \\ INRIA, Rennes, France \\ chuanyou.li@gmail.com
}

\author{
Michel Hurfin \\ INRIA, Rennes, France \\ michel.hurfin@inria.fr
}

\author{
Yun Wang \\ Southeast University, China \\ yunwang@seu.edu.cn
}

\author{
Lei Yu \\ Wuhan University, China \\ ly.wd@whu.edu.cn
}

\begin{abstract}
We consider the approximate consensus problem in a partially connected network of $n$ nodes where at most f nodes may suffer from Byzantine faults. We study under which conditions this problem can be solved using an iterative algorithm. A Byzantine node can equivocate: it may provide different values to its neighbors. To restrict the possibilities of equivocation, the 3-partial multicast primitive is considered. When a (correct or faulty) node uses this communication primitive, it provides necessarily the same value to the two identified receivers. Based on this communication primitive, a novel condition called f-resilient is proposed and proved to be necessary and sufficient to solve the approximate Byzantine consensus problem in a synchronous network. This condition takes into account two different communication primitives: unicast and 3-partial multicast. It expresses a trade-off between the two known approaches that make the problem solvable (increasing the number of neighbors or/and increasing the power of the communication primitives). The condition $f$ resilient does not require to eliminate all the possibilities of equivocation. Furthermore, it can be satisfied when there is just a majority of correct nodes. The relationships between the condition f-resilient and the condition h-disjoint (proposed by Alexander Jaffe et al. in 2012 to solve another problem, namely exact Byzantine consensus) are investigated. Two preliminary conclusions are obtained. When a network does not satisfy hdisjoint, it also does not satisfy f-resilient. But when a network satisfies h-disjoint, f-resilient is not necessarily satisfied. Finally, the condition is extended to cope with asynchronous networks.
\end{abstract}

Keywords-Approximate Consensus; Byzantine failure; Equivocation; Partial multicast; Iterative algorithm;

\section{INTRODUCTION}

The concept of approximate consensus was introduced by Dolev et al. [1]. In this problem, each node begins its participation by providing a real initial value. Then all the correct nodes must eventually obtain final values that $i$ ) are different from each other within a maximum gap denoted $\epsilon$ (convergence property) and $i$ ) must be in the range of initial values proposed by the correct nodes (validity property).

We consider approximate Byzantine consensus in a partially connected network of $n$ nodes where at most $f$ nodes may suffer from Byzantine faults. We study under which conditions this problem can be solved using an iterative algorithm [2], [3]. An iterative algorithm has two main advantages: it does not rely on message-relay and it does not assume a global knowledge. In particular, no node has to know the global network topology or the value of $n$. Based on iterative algorithms, [2] has proposed a necessary and sufficient condition related to the network topology which ensures the convergence property. Informally, the condition proposed in [2] imposes that each node has a sufficient number of neighbors. Moreover, in this solution, the maximal number of faulty nodes in the synchronous network is limited by the bound $n \geq 3 f+1$. This requirement on the proportion of faulty nodes is mainly due to the fact that a Byzantine node can equivocate, e.g., sends different messages to different neighbors. Stronger communication primitives such as the multicast has been considered to guarantee that an identical message is received by all the neighbors of a Byzantine node. The condition on the network topology proposed by [3] relies on the fact that all the possibilities of equivocation are eliminated: the bound becomes $n \geq 2 f+1$ in synchronous networks.

In this paper, in the particular context of the iterative approximate Byzantine consensus problem, we study the interest of restricting the possibilities of equivocation without suppressing all of them. To restrict the power of equivocation, we consider a basic communication primitive called a 3-partial multicast (also named a hyperedge in a communication topology). A 3-partial multicast identifies one sender and two receivers. When the three nodes use this primitive, the sender is forced to send identical messages to its two neighbors. The concept of hyperedges is strongly connected to the notion of uniform hyperedges [4]. Yet, while an uniform hyperedge of three nodes allows any of the three nodes to act as a sender or a receiver, such a symmetry does not exist in the case of a hyperedge where the unique sender is predefined. Based on partial multicast, we propose a new condition named $f$-resilient which is proved to be necessary and sufficient for reaching iterative approximate Byzantine consensus in a synchronous network. This condition is different from the conditions proposed in [2], [3]. First, rather than eliminating any possibility of equivocation like in [3], the $f$-resilient condition just restricts equivocation and requires only that $n \geq 2 f+1$. Second, the condition takes into account two communication primitives unicast and 3-partial multicast. Thus it allows to 
find a tradeoff between the two known approaches that make the problem solvable: increase the number of neighbors or/and increase the power of the communication primitives. This work has a strong connection with the $h$-disjoint condition proposed in [4] to solve another problem namely the exact Byzantine consensus problem. As the $h$-disjoint and the $f$-resilient conditions both refer to partial multicast primitives, we investigate the relationships between them. We show that when a network does not satisfy $h$-disjoint, it also does not satisfy $f$-resilient. But when a network satisfies $h$-disjoint, $f$-resilient is not always satisfied.

This paper is organized as follows. Section II introduces the model. Section III provides a formal definition of the iterative approximate consensus problem. Section IV sketches out some related works. In section $\mathrm{V}$, we define the condition $f$-resilient. Then we prove its necessity and sufficiency. In section VI, we investigate the relationships between $h$ disjoint and $f$-resilient. In section VII, we claim that $f$ resilient can be extended to cope with asynchronous systems.

\section{MODEL}

We consider a network composed of $n$ nodes whose identities are contained in the set $V=\left\{p_{1}, p_{2}, \ldots, p_{n}\right\}$. Some nodes (at most $f$ ) may suffer from Byzantine faults. These Byzantine nodes may stop their computation, behave arbitrarily, and even collude together. Therefore, $V$ is partitioned into two subsets denoted $V_{c}$ and $V_{b}$. The set $V_{c}$ contains the correct nodes which always follow the protocol specification. The Byzantine nodes belong to the set $V_{b}$ whose composition is not known by the correct nodes. By definition, $\left|V_{b}\right| \leq f$.

All the nodes communicate only by exchanging messages. In this study the communication process is assumed to be synchronous and no message is lost or modified during its transfer. We consider two different communication primitives: unicast or/and partial multicast. Unicast is corresponding to a point to point communication. A set $E_{U}$ contains all the unicast channels represented by ordered pairs. For example, $\left(p_{i} ; p_{j}\right) \in E_{U}$ implies that during the iterative computation, $p_{i}$ is expected to send periodically messages to $p_{j}$ using the unicast primitive. When a Byzantine node uses this communication primitive, it is able to equivocate. It can send simultaneously messages with different (fake or correct) values through different unicast channels. The use of a multicast primitive is intended to reduce the power of some Byzantine nodes. In this work, we consider a particular communication primitive called the 3-partial multicast. A 3-partial multicast channel is composed of a sender and two receivers. In the triplet of three nodes identified by such a multicast channel, the sender is forced to send simultaneously the same message to the two receivers. Consequently, the power of a Byzantine node is restricted when it uses a 3-partial multicast to send a message. A set $E_{M}$ contains all the triplets $\left(p_{i} ; p_{j}, p_{k}\right)$ such that, during the iterative computation, node $p_{i}$ is expected to send periodically messages to $p_{j}$ and $p_{k}$ using the 3-partial multicast primitive. The elements in $E_{M}$ are also called hyperedges (and not uniform hyperedges as the role of the sender is devoted to just one of the three nodes, namely the node identified on the left side of the semicolon in the triplet). Thus the fact that $\left(p_{i} ; p_{j}, p_{k}\right) \in E_{M}$ does not impose (nor prevent) that $\left(p_{j} ; p_{i}, p_{k}\right)$ or $\left(p_{k} ; p_{i}, p_{j}\right)$ also belongs to $E_{M}$. Receivers associated to a 3-partial multicast are not ordered: the notations $\left(p_{i} ; p_{j}, p_{k}\right)$ and $\left(p_{i} ; p_{k}, p_{j}\right)$ represent the same element. By definition, none of the two sets $E_{U}$ and $E_{M}$ contains duplicated elements. Like in [5], [6], [4], each correct node knows the (unicast or/and multicast) channels it has joined (either as a sender or a receiver). Even if this particular aspect is not discussed in this paper, the partial multicast model is motivated by various environments [7] (for example, a LAN like an Ethernet bus or a token ring, a group communication primitive, ...).

A static hybrid graph $G=\left(V, E_{U}, E_{M}\right)$ is used to represent the communication topology. This model is hybrid because it mixes information about the exploited two communication primitives (the set $E_{U}$ which models the use of the unicast primitive and the set $E_{M}$ which characterizes the use of the 3-partial multicast primitive). The hybrid graph is static because no mobility is considered and the choice of the communication primitives is supposed to be defined once and prior to the agreement protocol's execution. Based on the communication topology, we now introduce the concept source neighbor. Roughly, each node $p_{i}$ of $V$ is associated with a set denoted $N_{i}$ of source neighbors from which $p_{i}$ can receive messages. By definition, $p_{j} \in N_{i}$ if and only if either $\left(p_{j} ; p_{i}\right) \in E_{U}$ or $\left(p_{j} ; p_{i}, p_{k}\right) \in E_{M}$ ( $k$ identifies another node $p_{k}$ that is different from $p_{i}$ and $p_{j}$ ). Note that for $p_{i}$ and one of its source neighbor $p_{j}$, the set $E_{M}$ may contain several multicast channels associated to them. More precisely, the number of multicast channel $\left(p_{j} ; p_{i}, p_{k}\right)$ associated to $p_{j}$ and $p_{i}$ is comprised between 0 and $n-2$. For example, it could be the case both $\left(p_{j} ; p_{i}, p_{k_{1}}\right)$ and $\left(p_{j} ; p_{i}, p_{k_{2}}\right.$ ) (with $k_{1} \neq k_{2}$ ) belong to $E_{M}$. The network is not required to be fully connected: $N_{i}$ can be a proper subset of $V-\left\{p_{i}\right\}$. If some source neighbors of a correct node $p_{i}$ are Byzantine nodes, we assume that their power is limited: $p_{i}$ is able to identify the real sender of any received message. Thus a Byzantine node cannot use a fake identity to pretend to be another node.

\section{THE APPROXIMATE CONSENSUS PROBLEM}

In the approximate consensus problem, each correct node has an initial real value which will be continuously updated during the computation. An iterative solution that solves this problem assumes that each node executes a sequence of rounds. Each round is identified by a round number $r$ that belongs to the set $\mathfrak{R}=\{1,2, \ldots\}$. During a round, each correct node performs three operations: sending, receiving, 
and updating. More precisely, (1) a node sends its current value to its neighbors (using either unicast or multicast primitives); (2) it receives values from different source neighbors; (3) it updates its local value by using a function that takes into account its current value and some values received during the round. The value of a correct node $p_{i}$ when it begins the execution of round $r$ is denoted as $v_{i}(r)$. As a Byzantine node may propose different values during the same round, the notation $v_{i}(r)$ is meaningless if $p_{i}$ is not correct. By definition, the initial value of a correct node $p_{i}$ is denoted as $v_{i}(1)$. When round $r$ begins, among the set of $\left|V_{c}\right|$ values owned by the correct nodes, the minimum (respectively the maximum) value is denoted as $\min \left(V_{c}, r\right)$ (resp. $\max \left(V_{c}, r\right)$ ). More generally, at the beginning of a round $r$, if $S$ is a nonempty subset of $V_{c}$, the notation $\min (S, r)$ (resp. $\max (S, r)$ ) represents the minimum (resp. the maximum) value among the identified set of $|S|$ values.

Definition 1. The approximate Byzantine consensus problem is formally defined by two properties:

Validity: A correct node $p_{i}$ has always a valid value: $\forall r \in \mathfrak{R}, \forall p_{i} \in V_{c}, v_{i}(r) \in\left[\min \left(V_{c}, 1\right), \max \left(V_{c}, 1\right)\right]$.

Convergence: Eventually, all the correct nodes have values which are different from each other within a maximum predefined value $\epsilon$ (with $\epsilon>0$ ):

$\exists \tilde{r} \in \mathfrak{R}$ such that $\forall r>\tilde{r}, \max \left(V_{c}, r\right)-\min \left(V_{c}, r\right)<\epsilon$.

\section{RELATED WORKS}

Dolev et al. propose the earliest results on approximate Byzantine consensus [1]. When the network is fully connected and the total number of nodes is known, two algorithms [1] are proved correct respectively in a synchronous and an asynchronous network. [8], [9] investigate approximate Byzantine consensus in partially connected networks. However without message flooding, convergence cannot be reached. Based on the knowledge of the global topology, [10], [11] address the same problem and succeed to achieve the convergence. Without flooding and the knowledge of the global topology, [2] provides a solution based on an iterative algorithm. To ensure convergence, [2] proposes a sufficient and necessary condition to restrict the network topology. [12], [13] address the problem in a mobile environment and provide a sufficient and necessary condition which restricts the nodes' trajectories.

The strategy of some works [14], [3] consists in eliminating any possibility of equivocation. [14] improves the solution proposed in [1]: only $4 f+1$ nodes are needed in a fully connected asynchronous network. [3] considers iterative algorithms and propose a condition to ensure final convergence in partially connected networks. It requires $n \geq 2 f+1$ in synchronous networks. Rather than eliminating any possibility of equivocation, our condition only restricts them and requires $n \geq 2 f+1$ in synchronous networks.

Some works try to achieve similar goals but consider a different problem. In the case of the exact Byzantine consensus problem, [15], [16], [5], [6], [4] have studied the power of non-equivocation. By using transferable authentication and non-equivocation, [15] succeeds to transform any protocol that works under the crash model into a protocol that tolerates Byzantine failures without increasing the number of nodes. Based on the same methodology, in [16], the resiliency bound for asynchronous multiparty computation is improved to $n \geq 2 f+1$. Recently, uniform hyperedges [5], [6], [4] have been considered to restrict equivocation. [5] proposes a Byzantine consensus algorithm by considering there is an uniform hyperedge among every three nodes. However, [6], [4] point out that having uniform hyperedges everywhere is not necessary. To solve the exact Byzantine consensus problem, [4] proposes a sufficient and necessary condition on the network called $h$-disjoint. As this work is strongly related to our study (even if the targeted problems are different), we investigate the relationships between the conditions $h$-disjoint and $f$-resilient in section VI.

\section{A NECESSARY AND SUFFICIENT CONDITION}

\section{A. The proposed condition}

To define formally our f-resilient condition, we first introduce the notion of safe $\digamma$ partition (similar to[2]). Recall that $\left\langle S_{1}, S_{2}, \ldots, S_{m}\right\rangle$ is a partition of a set $S$, if and only if, $S_{i} \cap S_{j}=\emptyset(i \neq j)$ and $\bigcup_{1 \leq i \leq m} S_{i}=S$.

Definition 2. Let $G=\left(V, E_{U}, E_{M}\right)$ be an hybrid-graph, $\langle F, L, M, R\rangle$ is a $\digamma$ partition of $G$ if and only if it is a partition of $V,|F| \leq f,|L|>0,|M| \geq 0$ and $|R|>0$.

Let $\langle F, L, M, R\rangle$ be a $\digamma$ partition of $G$. We use the notation $\mathbb{L}$ to represent the set $L \cup M$, while $\mathbb{R}$ corresponds to $R \cup M$. To determine if a $\digamma$ partition is safe or not, we focus on all the pairs of nodes $\left(p_{i}, p_{j}\right)$ such that $p_{i} \in L$ and $p_{j} \in R$. For each pair, we consider the source neighbors of $p_{i}$ which belong either to $M$ or $R$ (i.e., $N_{i} \cap \mathbb{R}$ ) and the source neighbors of $p_{j}$ which belong either to $L$ or $M$ (i.e., $\left.N_{j} \cap \mathbb{L}\right)$. We also consider all the hyperedges of $E_{M}$ where $p_{i}$ and $p_{j}$ act as the two receivers while the sender is a node $p_{x}$ belonging to $F$. A subset $F_{i j}$ of $F$ is defined as follows: $F_{i j}=\left\{p_{x} \mid p_{x} \in F \wedge\left(p_{x} ; p_{i}, p_{j}\right) \in E_{M}\right\}$.

Definition 3. $\langle F, L, M, R\rangle$ is a safe $\digamma$ partition of $G$ if and only if one of the two following properties holds.

$C_{1}: \exists p_{i} \in L$ such that $\left|N_{i} \cap \mathbb{R}\right| \geq f+1$ or $\exists p_{j} \in R$ such that $\left|N_{j} \cap \mathbb{L}\right| \geq f+1$;

$C_{2}: \exists p_{i} \in L, \exists p_{j} \in R$, such that $1 \leq\left|N_{i} \cap \mathbb{R}\right| \leq f$, $1 \leq\left|N_{j} \cap \mathbb{L}\right| \leq f,\left|F_{i j}\right|+\left|N_{i} \cap \mathbb{R}\right|+\left|N_{j} \cap \mathbb{L}\right| \geq 2 f+1$.

Examples of safe $\digamma$ partition are depicted in Figure 1. In Definition 3, the two properties $C_{1}$ and $C_{2}$ are exclusive. Both can be false but when one is true, the other one is false. Property $C_{1}$ expresses a requirement on the communication topology. At least one node in $L$ (or in $R$ ) must have a sufficient number of source neighbors in $\mathbb{R}$ (or in $\mathbb{L}$ ). Without the property $C_{2}$, our condition is exactly the one 


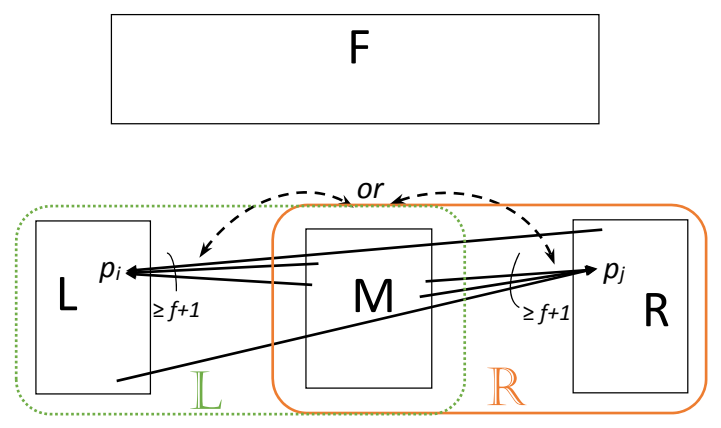

(a) An example of $C_{1}$

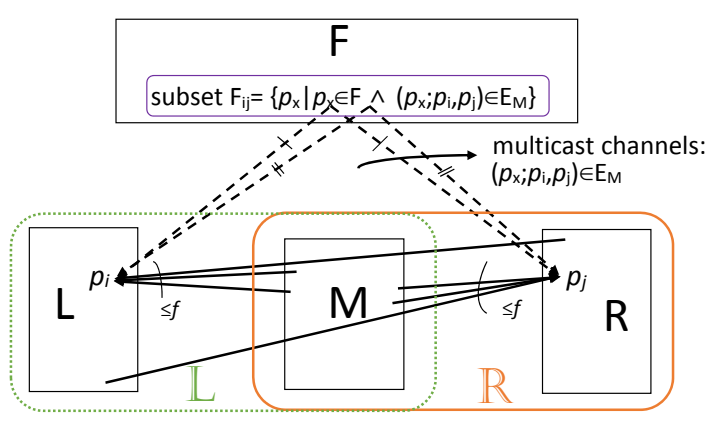

(b) An example of $C_{2}$

Figure 1. Examples of safe $\digamma$ partitions

Table I

EXAMPLE OF $f$-RESILIENT, $n=5, f=2$

\begin{tabular}{|c|c|c|c|c|c|}
\hline sender & $p_{a}$ & $p_{b}$ & $p_{c}$ & $p_{d}$ & $p_{e}$ \\
\hline \multirow{5}{*}{ multicast channels } & $\left(p_{a} ; p_{b}, p_{d}\right)$ & $\left(p_{b} ; p_{c}, p_{e}\right)$ & $\left(p_{c} ; p_{d}, p_{a}\right)$ & $\left(p_{d} ; p_{e}, p_{b}\right)$ & $\left(p_{e} ; p_{b}, p_{d}\right)$ \\
\cline { 2 - 6 } & $\left(p_{a} ; p_{b}, p_{c}\right)$ & $\left(p_{b} ; p_{c}, p_{d}\right)$ & $\left(p_{c} ; p_{d}, p_{e}\right)$ & $\left(p_{d} ; p_{e}, p_{a}\right)$ & $\left(p_{e} ; p_{b}, p_{c}\right)$ \\
\cline { 2 - 6 } & $\left(p_{a} ; p_{b}, p_{e}\right)$ & $\left(p_{b} ; p_{d}, p_{a}\right)$ & $\left(p_{c} ; p_{b}, p_{e}\right)$ & $\left(p_{d} ; p_{a}, p_{c}\right)$ & $\left(p_{e} ; p_{a}, p_{c}\right)$ \\
\cline { 2 - 6 } & $\left(p_{a} ; p_{c}, p_{e}\right)$ & $\left(p_{b} ; p_{d}, p_{e}\right)$ & $\left(p_{c} ; p_{e}, p_{a}\right)$ & $\left(p_{d} ; p_{c}, p_{b}\right)$ & $\left(p_{e} ; p_{c}, p_{d}\right)$ \\
\cline { 2 - 6 } & $\left(p_{a} ; p_{c}, p_{d}\right)$ & $\left(p_{b} ; p_{a}, p_{c}\right)$ & $\left(p_{c} ; p_{d}, p_{b}\right)$ & $\left(p_{d} ; p_{e}, p_{c}\right)$ & $\left(p_{e} ; p_{d}, p_{a}\right)$ \\
\hline
\end{tabular}

proposed in [2]. Thus, property $C_{2}$ extends the work of [2] when the topological requirement is not satisfied. In fact, property $C_{2}$ captures the interest of using a multicast primitive to prevent equivocation. The fact that the number of source neighbors is not sufficient is counterbalanced by the existence of hyperedges that link a sender $p_{x}$ of $F$ to a receiver $p_{i}$ of $L$ and a receiver $p_{j}$ of $R$. The number of such hyperedges, namely $\left|F_{i j}\right|$, the number of source neighbors of $p_{i}$ in $\mathbb{R}$, namely $\left|N_{i} \cap \mathbb{R}\right|$, and the number of source neighbors of $p_{j}$ in $\mathbb{L}$, namely $\left|N_{j} \cap \mathbb{L}\right|$ are added. The sum has to be greater than or equal to $2 f+1$.

Definition 4. A hybrid-graph $G$ is named f-resilient if and only if all its $\digamma$ partitions are safe.

The two properties that characterize a safe $\digamma$ partition allow to find a tradeoff between two approaches: increasing the connectivity (more source neighbors) or/and increasing the use of powerful communication primitives (more hyperedges). Through an example (See Table I), we show that an hybrid graph $G$ can be $f$-resilient even if all the possibilities of equivocation are not eliminated.

In this example, $G$ is composed of five nodes $p_{a}, p_{b}$, $p_{c}, p_{d}, p_{e}$ among which at most two can be Byzantine nodes $(f=2)$. Any $\digamma$ partition such that $|F|=2$ and $|M|=0$ can not satisfy the first property that characterizes a safe partition: neither $L$ nor $R$ can contain more than two elements and thus the limit $f+1=3$ imposed by the first property cannot be reached. The use of hyperedges is mandatory. Table I identifies a solution where 25 hyperedges are used (among the 30 which could be defined). In this solution, $G$ is $f$-resilient even if equivocation is restricted but not completely eradicated: if $p_{a}$ is a Byzantine node, it can send different messages to $p_{d}$ and $p_{e}$. This example also illustrates that the $f$-resilient condition requires $n \geq 2 f+1$. Obviously, when $n=2 f=4$, any $\digamma$ partition with $|F|=2,|L|=1,|M|=0$ and $|R|=1$ can not be safe due to the fact that $|F|+|L|+|R|<2 f+1$.

As the condition $f$-resilient requires that all $\digamma$ partitions are safe, an interesting future work consists in investigating the relationships between $f$-resilient and the connectivity of the directed communication topology. Herein we only point out that when $n=2 f+1$, the condition implies that the communication topology is fully connected. However, when $n>2 f+1$, this is not necessarily required.

\section{B. Necessity}

In this section, we prove the necessity of $f$-resilient.

Lemma 1. No iterative algorithm solves the approximate Byzantine consensus, if $G$ is not $f$-resilient.

Proof: Assume that an iterative algorithm (executed endlessly by every correct node) is able to solve the approximate Byzantine consensus problem. The validity and convergence properties that characterize this problem have to be satisfied by this algorithm in any scenarios. To show that this assumption leads to a contradiction, we will identify a particular scenario denoted $S$ in which, after a round $r$, at least two correct nodes (denoted $p_{x}$ and $p_{y}$ ) definitely stop the convergence process because they have to ensure the validity property is never violated. More precisely, during 
any round $r^{\prime}$ such that $r^{\prime} \geq r, p_{x}$ (resp. $p_{y}$ ) does not change its value $v_{x}=\min \left(V_{c}, r\right)\left(\right.$ resp. $\left.v_{y}=\max \left(V_{c}, r\right)\right)$. Consequently the difference $v_{y}-v_{x}$ remains equal to a value greater than $\epsilon$. In the scenario $S$, the node $p_{k}$ (with $k=x$ or $k=y$ ) can receive at most $2 f$ values during a round. Among these values, at most $f$ values (that have been provided by nodes from a set $A_{k}$ ) are smaller or equal to its own value $v_{k}$ and at most $f$ values (that have been provided by nodes from a set $B_{k}$ ) are greater or equal to $v_{k}$. As $A_{k} \cap B_{k}=\emptyset$, the proof relies on the fact that the correct node $p_{k}$ can not know if a node is correct or not. It cannot distinguish between two extreme situations: 1) $A_{k} \subseteq V_{c}$ and $B_{k} \subseteq V_{b}$, 2) $A_{k} \subseteq V_{b}$ and $B_{k} \subseteq V_{c}$. If the node $p_{k}$ computes a new value $v$ during a round $r^{\prime} \geq r$, this value $v$ must satisfy the property $v \leq v_{k}$ (because the first possible situation can be the actual scenario) and the property $v \geq v_{k}$ (because the second possible situation can be the actual scenario). Thus, to ensure the validity property, the algorithm must leave the values of $p_{x}$ and $p_{y}$ unchanged after round $r$. Consequently it violates the convergence property.

Above, we have depicted the general structure of the proof. Now, we demonstrate the existence of the nodes $p_{x}$ and $p_{y}$ and we identify a scenario $S$ for which four sets $A_{x}$, $B_{x}, A_{y}$ and $B_{y}$ can be defined with respect to $p_{x}$ and $p_{y}$. Of course, the following requirements have to be satisfied: $A_{x} \cap B_{x}=\emptyset, A_{y} \cap B_{y}=\emptyset$, the cardinalities of these four sets are less or equal to $f$ and the values provided by the nodes of $A_{x}$ (resp. $A_{y}$ ) are smaller or equal to $v_{x}$ (resp. $v_{y}$ ) while the values provided by the nodes of $B_{x}$ (resp. $B_{y}$ ) are greater or equal to $v_{x}$ (resp. $v_{y}$ ).

As the hybrid-graph $G$ is not $f$-resilient, there exists at least one unsafe $\digamma$ partition denoted $\left\langle F^{\prime}, L^{\prime}, M^{\prime}, R^{\prime}\right\rangle$ such that: $\forall p_{i} \in L^{\prime}, \forall p_{j} \in R^{\prime},\left|N_{i} \cap \mathbb{R}^{\prime}\right| \leq f,\left|N_{j} \cap \mathbb{L}^{\prime}\right| \leq f$ and $\left|F_{i j}^{\prime}\right|+\left|N_{i} \cap \mathbb{R}^{\prime}\right|+\left|N_{j} \cap \mathbb{L}^{\prime}\right| \leq 2 f$. Furthermore, we have $\left|F^{\prime}\right| \leq f,\left|L^{\prime}\right|>0$ and $\left|R^{\prime}\right|>0$. The scenario $S$ is based on this unsafe partition. We assume that the set $F^{\prime}$ is corresponding to the set of Byzantine nodes $V_{b}$. Consequently, all the nodes of $L^{\prime} \cup M^{\prime} \cup R^{\prime}$ are correct. At the beginning of a round $r$ (possibly the first round), we assume also that all the nodes of $L^{\prime}$ have the minimum value $\min \left(V_{c}, r\right)$ and all the nodes of $R^{\prime}$ have the maximum value $\max \left(V_{c}, r\right)$. Note that the remaining correct nodes of $M^{\prime}$ (if any) have their values within the range $\left[\min \left(V_{c}, r\right), \max \left(V_{c}, r\right)\right]$. Thereafter we consider that $p_{x}$ is any node of $L^{\prime}$ while $p_{y}$ is any node of $R^{\prime}$. As $\left|L^{\prime}\right|>0$ and $\left|R^{\prime}\right|>0$, two such nodes always exist and, by definition, $v_{x}=\min \left(V_{c}, r\right)$ while $v_{y}=\max \left(V_{c}, r\right)$. Regarding the behaviors of the Byzantine nodes in scenario $S$, we consider that the set $F^{\prime}$ is partitioned into three subsets denoted $F^{1}, F^{2}$, and $F^{3}$. During each round, Byzantine nodes of $F^{1} \cup F^{2}=F_{x y}^{\prime}$ use the multicast primitive and always provide the same value to $p_{x}$ and $p_{y}$ while Byzantine nodes of $F^{3}=F^{\prime} / F_{x y}^{\prime}$ equivocate and provide different values. The nodes of $F^{1}$ always provide a value belonging to the range $\left(-\infty, v_{x}\right)$.
The nodes of $F^{2}$ always provide a value belonging to the range $\left(v_{y},+\infty\right)$. The nodes of $F^{3}$ always provide $p_{x}$ a value belonging to the range $\left(-\infty, v_{x}\right)$ and always provide $p_{y}$ a value belonging to the range $\left(v_{y},+\infty\right)$. The scenario $S$ is unique but nevertheless the cardinality of $F^{2}$ depends on a characteristic of the unsafe partition: if $\left|F_{x y}^{\prime}\right|+\left|N_{y} \cap \mathbb{L}^{\prime}\right| \leq f$ then $\left|F^{2}\right|=0$ else $\left|F^{2}\right|=\left|F_{x y}^{\prime}\right|+\left|N_{y} \cap \mathbb{L}^{\prime}\right|-f$. Consequently, we have always $\left|F^{1}\right|+\left|N_{y} \cap \mathbb{L}^{\prime}\right| \leq f$. Furthermore, as $\left|F_{x y}^{\prime}\right|+\left|N_{x} \cap \mathbb{R}^{\prime}\right|+\left|N_{y} \cap \mathbb{L}^{\prime}\right| \leq 2 \bar{f}$, we have also $\left|F^{2}\right|+\left|N_{x} \cap \mathbb{R}^{\prime}\right| \leq f$.

Let $A_{x}=F^{1} \cup F^{3}, B_{x}=F^{2}+\left|N_{x} \cap \mathbb{R}^{\prime}\right|, B_{y}=F^{2} \cup F^{3}$ and $A_{y}=F^{1}+\left|N_{y} \cap \mathbb{L}^{\prime}\right|$. These sets satisfy all the specified requirements and thus $S$ is a particular scenario where the convergence property cannot not be ensured (without the risk of violating the validity property). Note that during the computation, nodes of $M^{\prime}$ can change their values but this will have no consequence for $p_{x}$ and $p_{y}$.

\section{Sufficiency}

To prove that the condition $f$-resilient is also sufficient, we propose and describe an iterative algorithm called LIABC (for Linear Iterative Approximate Byzantine Consensus) that satisfies the validity property. Then, assuming that the $f$ resilient condition is satisfied, we prove that the LIABC algorithm ensures the convergence property.

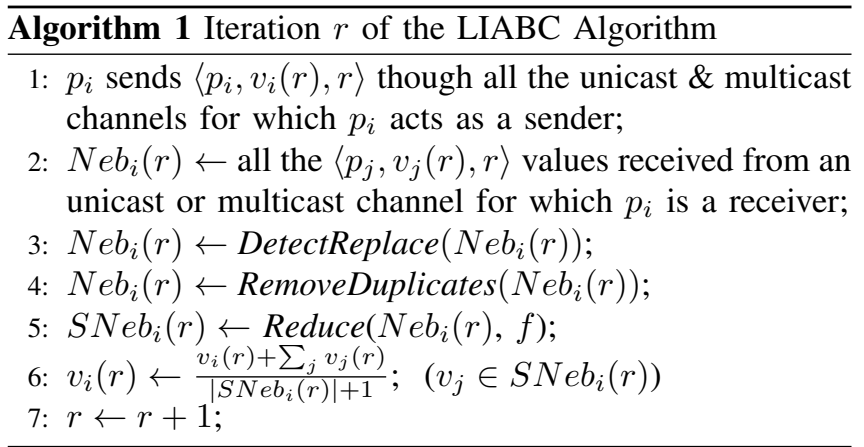

1) The LIABC algorithm and its validity: The pseudo code in Figure 1 describes the behavior of a correct node $p_{i}$ during an iteration $r$. During the initialization phase (not described in Figure1), $r$ is set to the value 1 and the initial value of $p_{i}$ is stored in the variable $v_{i}(1)$. During the sending step of an iteration $r$ (line 1), for each channel in which $p_{i}$ acts as a sender (i.e. unicast channels $\left(p_{i} ; ?\right)$ and multicast channels $\left.\left(p_{i} ; ?, ?\right)\right) p_{i}$ sends a message with its identity $p_{i}$, its current value $v_{i}(r)$ and the round number $r$. During the receiving step (line 2), $p_{i}$ waits to receive messages coming from channels for which it acts as a receiver (i.e. unicast channels $\left(? ; p_{i}\right)$ and multicast channels $\left(? ; p_{i}, ?\right)$ or $\left.\left(? ; ?, p_{i}\right)\right)$. The set $N e b_{i}(r)$ contains all the received messages. During the updating step (lines 3-6), the node $p_{i}$ first calls the function DetectReplace to identify some Byzantine nodes among its neighbors. If a node $p_{b}$ shares 
a communication channel with $p_{i}$ and has not sent the expected message, $p_{b}$ is necessarily a Byzantine node. Indeed, the network is synchronous and no channel drops messages. Similarly, if $p_{i}$ shares several channels with a same source neighbor $p_{b}$ but receives different values from $p_{b}$ during the same round, $p_{i}$ can also conclude that $p_{b}$ is a Byzantine node. Of course, only unexpected behaviors are detected and thus some Byzantine nodes remains undetected: the failure detection mechanism satisfies a strong accuracy property (no correct node is ever suspected) but no completeness property. If a Byzantine node $p_{b}$ is detected during round $r$, all the information it has provided is removed from the set $N e b_{i}(r)$. Finally, for each detected node $p_{b}$, the function DetectReplace insert a single information, namely $\left\langle p_{b}, \perp, r\right\rangle$ in $N e b_{i}(r)$. The value $\perp$ is such that any value provided by a correct node is strictly greater than $\perp$. Note that the code can be optimized to ignore the values provided by detected nodes during the remainder of computation and not only during the current iteration. Herein, we ignore this possibility. As a node (even a correct one) can send several messages to $p_{i}$ (through different channels), the set $N e b_{i}(r)$ can contain duplicated values. The call to the function RemoveDuplicates (line 5) ensures that $N e b_{i}(r)$ contains only one value from each source neighbor. Finally, the call to the function Reduce aims at suppressing some values to keep only those that are not risky (i.e. using the remaining values during the computation of the new value of $p_{i}$ cannot compromise the validity property). If $N e b_{i}(r)$ contains less than $f$ values greater than $v_{i}(r), p_{i}$ suppresses all these values. Otherwise, $p_{i}$ suppresses the $f$ largest values of $N e b_{i}(r)$. Likewise, if $N e b_{i}(r)$ contains less than $f$ values smaller than $v_{i}(r), p_{i}$ suppresses all these values. Otherwise, $p_{i}$ suppresses the $f$ smallest values. Note that at most $2 f$ values are suppressed. Obviously, the $\perp$ values corresponding to detected Byzantine nodes are suppressed at this stage. After reducing, $p_{i}$ calculates the average between its current value $v_{i}(r)$ and the remaining values of $S N e b_{i}(r)$ (line 6). The round number $r$ is increased by 1 at the end.

\section{Theorem 1. The algorithm LIABC satisfies validity.}

Of course, the validity property holds after the initialization phase. Let us consider that this is no more true when round $r>1$ begins. It means that, during round $r-1$, at least one correct node $p_{x}$ has used a value beyond the range $\left[\min \left(V_{c}, 1\right), \max \left(V_{c}, 1\right)\right]$ to update its own value. The function Reduce ensures that this scenario is impossible: if a value $v$ proposed by a undetected Byzantine node still remains in $S N e b_{x}(r-1)$, then at least one correct neighbor of $p_{x}$ (or $p_{x}$ itself) has provided a value $v_{a}$ and at least one correct neighbor of $p_{x}$ (or $p_{x}$ itself) has provided a value $v_{b}$ such that $v_{a} \leq v \leq v_{b}$. Thus $p_{x}$ can not compute an average (line 6) with a value beyond the range $\left[\min \left(V_{c}, r-1\right), \max \left(V_{c}, r-1\right)\right]$. Indeed the algorithm LIABC ensures a stronger property (Theorem 2 ).
Theorem 2. During the sequence of rounds $1 \ldots r \ldots$, the minimum value $\min \left(V_{c}, r\right)$ is non-decreasing and the maximum value $\max \left(V_{c}, r\right)$ is non-increasing.

2) The convergence: Definition 3 identifies two distinct safety properties (denoted $C_{1}$ and $C_{2}$ ). Therefore, when an hybrid graph $G$ is $f$-resilient, two cases can be distinguished. First, all the $\digamma$ partitions are safe because each of them satisfies the property $C_{1}$. Second, some (at least one) $\digamma$ partitions are safe because they satisfy the property $C_{2}$. In [2], the authors have already consider the first case. So, we only focus on the proof related to the second case. Any $\digamma$ partition mentioned below is supposed to be safe. A particular set of partitions $\Gamma$ is defined.

Definition 5. $\Gamma=\left\{\digamma=\langle F, L, M, R\rangle \mid F=V_{b}\right\}$

Lemma 2. Let $G$ be a $f$-resilient hybrid graph and let $\digamma$ be a partition of $\Gamma$ such that, during a round $r, \max (L, r)<$ $\min (\mathbb{R}, r)$ and $\max (\mathbb{L}, r)<\min (R, r)$. Whatever the behaviors of the Byzantine nodes of set $F$, at least one of the two following properties holds:

1) $\exists p_{i} \in L, \exists v \in S N e b_{i}(r)$ s.t. $v \in[\min (\mathbb{R}, r), \max (\mathbb{R}, r)]$ 2) $\exists p_{j} \in R, \exists v \in S N e b_{j}(r)$ s.t. $v \in[\min (\mathbb{L}, r), \max (\mathbb{L}, r)]$

Proof: The proof is by contradiction. Consider a particular $\digamma$ partition $\langle F, L, M, R\rangle$ of the set $\Gamma$ such that $\max (L, r)<\min (\mathbb{R}, r)$ and $\max (\mathbb{L}, r)<\min (R, r)$. Assume that this partition satisfies none of the dual properties.

As $G$ is $f$-resilient, the partition $\digamma$ is safe. Yet the property $C_{1}$ cannot be satisfied by this partition: $\forall p_{i} \in L$ and $\forall p_{j} \in$ $R$, neither $\left|N_{i} \cap \mathbb{R}\right| \geq f+1$ nor $\left|N_{j} \cap \mathbb{L}\right| \geq f+1$ is possible. As the proofs are symmetric, we just show why $\left|N_{i} \cap \mathbb{R}\right| \geq f+1$ is impossible. As $\max (L, r)<\min (\mathbb{R}, r)$, if $\left|N_{i} \cap \mathbb{R}\right| \geq f+1$, whatever the values received from the Byzantine nodes, $p_{i}$ gathers at least $f+1$ values from correct nodes of $\mathbb{R}$. Thus after reducing (line 5$), S N e b_{i}(r)$ contains at least a value $v$ within the range $[\min (\mathbb{R}, r), \max (\mathbb{R}, r)]$.

Therefore, the property $C_{2}$ is necessarily satisfied by $\digamma$ : $\exists p_{i} \in L, \exists p_{j} \in R$, such that $1 \leq\left|N_{i} \cap \mathbb{R}\right| \leq f, 1 \leq$ $\left|N_{j} \cap \mathbb{L}\right| \leq f$ and $\left|F_{i j}\right|+\left|N_{i} \cap \mathbb{R}\right|+\left|N_{j} \cap \mathbb{L}\right| \geq 2 f+1$. The $\left|N_{i} \cap \mathbb{R}\right|$ correct nodes that belong to $\mathbb{R}$ provide to $p_{i}$ a value $v$ such that $v \geq \min (\mathbb{R}, r)$. The node $p_{i}$ can also receive values from the Byzantine nodes of $F$. Let us define two subsets of $F$ denoted $F^{1}$ and $F^{2}$ such that $F^{1}$ contains all the nodes of $F$ that send to $p_{i}$ a value $v^{1}$ such that $v^{1} \geq \min (\mathbb{R}, r)$ and $F^{2}$ contains all the nodes of $F$ that send to $p_{i}$ a value $v^{2}$ such that $v^{2}<\min (\mathbb{R}, r)$. We must have $\left|F^{1}\right|+\left|N_{i} \cap \mathbb{R}\right| \leq f$. Otherwise, after the reducing operation, a value within the range $[\min (\mathbb{R}, r), \max (\mathbb{R}, r)]$ will still remain in the set $S N e b_{i}(r)$ of $p_{i}$.

Consider now the node $p_{j}$. This node receives from $\mid N_{j} \cap$ $\mathbb{L} \mid$ correct nodes of $\mathbb{L}$ a value $v$ such that $v \leq \max (\mathbb{L}, r)$. As we have $\left|N_{j} \cap \mathbb{L}\right| \leq f$ and $\left|F_{i j}\right|+\left|N_{i} \cap \mathbb{R}\right|+\left|N_{j} \cap \mathbb{L}\right| \geq 2 f+1$, we can conclude that $\left|F_{i j}\right|+\left|N_{i} \cap \mathbb{R}\right| \geq f+1$. We have previously shown that $\left|F^{1}\right|+\left|N_{i} \cap \mathbb{R}\right| \leq f$. Consequently, 
$\left|F_{i j}\right|>\left|F^{1}\right|$. Byzantine nodes of $F_{i j}$ can either provide to $p_{j}$ the value they also provide to $p_{i}$ or provide no value to $p_{j}$. In the latter case, the node is recognized as been a Byzantine node by $p_{j}$ which will add a $\perp$ value (such that $\perp<\min (\mathbb{L}, r)<\min (\mathbb{R}, r))$ to its set $N e b_{j}(r)$ (line 3). Consequently, among the nodes of $F^{2}$, at least $\left|F_{i j}\right|-\left|F^{1}\right|$ Byzantine nodes also send to $p_{j}$ a value $v^{2}$ such that $v^{2}<\min (\mathbb{R}, r)$. If $M$ is empty, any value $v^{2}$ such that $v^{2}<\min (\mathbb{R}, r)$ satisfies also $v^{2} \leq(\mathbb{L}, r)$. Otherwise, when the set $M$ is not empty, we have $\min (\mathbb{R}, r) \leq \max (\mathbb{L}, r)$. Consequently, $p_{j}$ can receive $\left|F_{i j}\right|-\left|F^{1}\right|+\left|N_{j} \cap \mathbb{L}\right|$ values that are less or equal to $\max (\mathbb{L}, r)$. Again, as we have previously shown that $\left|F^{1}\right|+\left|N_{i} \cap \mathbb{R}\right| \leq f$, the inequality $\left|F_{i j}\right|+\left|N_{i} \cap \mathbb{R}\right|+\left|N_{j} \cap \mathbb{L}\right| \geq 2 f+1$ leads us to conclude that $\left|F_{i j}\right|-\left|F^{1}\right|+\left|N_{j} \cap \mathbb{L}\right| \geq f+1$. After the call to Reduce, the $f$ minimum values are suppressed. But at least one value $v$ within the range $v \in[\min (\mathbb{L}, r), \max (\mathbb{L}, r)]$ still remains. This contradicts our assumption.

For each round $r$, we define now two particular sets of nodes as follows. The set $S_{\min }(r)$ includes all the correct nodes that have the minimum value $\min \left(V_{c}, r\right)$ at the beginning of round $r$ while the set $S_{\max }(r)$ includes all the correct nodes that have the maximum value $\max \left(V_{c}, r\right)$.

Corollary 1. Let $G$ be a f-resilient hybrid graph. For any round $r$, at least one of the two following properties holds:

1) $\exists p_{i} \in S_{\min }(r)$, such that $v_{i}(r+1)>\min \left(V_{c}, r\right)$

2) $\exists p_{j} \in S_{\max }(r)$, such that $v_{j}(r+1)<\max \left(V_{c}, r\right)$

Proof: During round $r$, consider the partition $\langle F, L, M, R\rangle$ of $\Gamma$ that satisfies $L=S_{\min }(r)$ and $R=$ $S_{\max }(r)$. The conditions expressed in Lemma 2 hold and consequently either a node $p_{i}$ of $S_{\min }(r)$ computes (at line 6) an average with a value greater than $\min \left(V_{c}, r\right)$ or a node $p_{j}$ of $S_{\max }(r)$ computes an average with a value less than $\max \left(V_{c}, r\right)$. Thus, in round $r+1$, either $v_{i}(r+1)>\min \left(V_{c}, r\right)$ or $v_{j}(r+1)<\max \left(V_{c}, r\right)$.

Corollary 2. $\forall r \in \mathfrak{R}$, there always exists a round $r^{*}$ with $r^{*}>r$, such that $\forall r^{\prime} \geq r^{*}, \max \left(V_{c}, r^{\prime}\right)-\min \left(V_{c}, r^{\prime}\right)<$ $\max \left(V_{c}, r\right)-\min \left(V_{c}, r\right)$.

Proof: Theorem 2 guarantees $\min \left(V_{c}, r\right)$ is nondecreasing and $\max \left(V_{c}, r\right)$ is non-increasing. Remember that the Reduce function ensures that no correct node computes a new value with a value outside the range $\left[\min \left(V_{c}, r\right), \max \left(V_{c}, r\right)\right]$. Therefore, if a correct node holds a value within the range $\left(\min \left(V_{c}, r\right), \max \left(V_{c}, r\right)\right)$ in round $r$, by using average calculation (line 6), its value cannot reach the bounds $\min \left(V_{c}, r\right)$ or $\max \left(V_{c}, r\right)$ in the future. It means that both $\left|S_{\min }(r)\right|$ and $\left|S_{\max }(r)\right|$ are non-increasing. Corollary 1 ensures that in round $r$, either a correct node that has the minimum value $\min \left(V_{c}, r\right)$ will increase its value or a correct node that has the maximum value will decrease its value. As both $\left|S_{\min }(r)\right|$ and $\left|S_{\max }(r)\right|$ are limited, there must exist a round $r^{*}$, such that $\forall r^{\prime} \geq r^{*}$ either $\min \left(V_{c}, r^{\prime}\right)>\min \left(V_{c}, r\right)$ or $\max \left(V_{c}, r^{\prime}\right)<\max \left(V_{c}, r\right)$.

Lemma 3. The algorithm LIABC can solve approximate Byzantine consensus in $G$ if $G$ is $f$-resilient.

Corollary 2 ensures the distance $\max \left(V_{c}, r\right)-\min \left(V_{c}, r\right)$ decreases continuously. Furthermore, due to Theorem 2, any decrease of this distance is irreversible. To prove lemma 3, we have still to demonstrate that, when $G$ is $f$-resilient, for any given $\epsilon>0$, there does not exist a value $\mu \geq \epsilon$, such that $\lim _{r \rightarrow+\infty} \max \left(V_{c}, r\right)-\min \left(V_{c}, r\right)=\mu$. We prove this by contradiction. First we define Hypothesis 1 and then we prove it is not true.

Hypothesis 1. Given a value $\epsilon>0$, there exists a value $\mu \geq \epsilon$, such that $\lim _{r \rightarrow+\infty} \max \left(V_{c}, r\right)-\min \left(V_{c}, r\right)=\mu$.

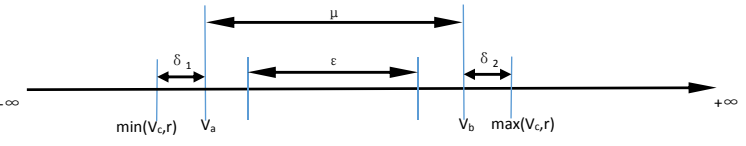

Figure 2. The example of $\epsilon$ and $\mu$

To facilitate the proof, we rewrite Hypothesis 1 in an equivalent way. There exist two values denoted $v_{a}$ and $v_{b}$ with $v_{b}-v_{a}=\mu$ (see Figure 2). For any round $r$, we suppose that $\min \left(V_{c}, r\right)=v_{a}-\delta_{1}(r)$ and $\max \left(V_{c}, r\right)=$ $v_{b}+\delta_{2}(r)$. The dual parameters $\delta_{1}(r)$ and $\delta_{2}(r)$ satisfy $\delta_{1}(r) \geq 0$ and $\delta_{2}(r) \geq 0$ but at least one of them is strictly greater than 0 (no violation of Corollary 2). If $\delta_{1}(r)>0$ (or/and $\delta_{2}(r)>0$ ), as $r$ increases $\delta_{1}(r)$ (or/and $\delta_{2}(r)$ ) is monotone non-increasing and converges to zero. Based on these new notations, we define two sets related to $r$ : $S_{n \min }(r)=\left\{p_{i} \mid v_{i}(r) \in\left[v_{a}-\delta_{1}(r), v_{a}\right] \wedge p_{i} \in V_{c}\right\}$ and $S_{n \max }(r)=\left\{p_{i} \mid v_{i}(r) \in\left[v_{b}, v_{b}+\delta_{2}(r)\right] \wedge p_{i} \in V_{c}\right\}$. $S_{n \text { min }}(r)$ includes the correct nodes that have a value less than or equal to $v_{a}$ in round $r$, while $S_{n \max }(r)$ includes the correct nodes that have a value greater than or equal to $v_{b}$ in round $r$. Note that by assumption $S_{n \min }(r) \neq \emptyset$ and $S_{n \max }(r) \neq \emptyset$ in any round $r$.

Lemma 4. Suppose Hypothesis 1 is true. If $\delta_{1}(r)>0$ in any round $r$, the set $V_{c}$ has a proper subset $V_{c}^{\prime} \neq \emptyset$, such that $\forall p_{i} \in V_{c}^{\prime}$ its value $v_{i}$ converges to $v_{a}$. Symmetrically, if $\delta_{2}(r)>0$ in any round $r$, the set $V_{c}$ has a proper subset $V_{c}^{\prime \prime} \neq \emptyset$, such that $\forall p_{j} \in V_{c}^{\prime \prime}$ its value $v_{j}$ converges to $v_{b}$.

Proof: As the proofs for $V_{c}^{\prime}$ and $V_{c}^{\prime \prime}$ are similar, we only consider the case of $V_{c}^{\prime}$. First, if $V_{c}^{\prime}$ exists (nonempty), it is a proper subset of $V_{c}$. Otherwise, it means that $S_{n \max }$ will become empty during a given round and thus Hypothesis 1 is not true. The proof focuses now on the correct nodes $p_{i}$ that stay in $S_{n \min }$ forever or that leave and integrate again this set infinitely often. These nodes are elements of a set $\tilde{V}_{c}$. More formally, $\tilde{V}_{c} \subseteq V_{c}$ and $\forall p_{i} \in \tilde{V}_{c}$ there exists an infinite subset $\mathfrak{R}_{i} \subset \Re$, such that $\forall r \in \Re_{i}, p_{i} \in S_{n \min }(r)$ and $\forall r \notin$ 
$\Re_{i}, p_{i} \notin S_{n m i n}(r)$. By Hypothesis 1 , we know $S_{n m i n}(r)$ is never empty. Consequently, $\tilde{V}_{c}$ is also non empty. If we prove that the value $v_{i}(r)$ of each node $p_{i}$ of $\tilde{V}_{c}$ converges to $v_{a}$, we can conclude the existence of $V_{c}^{\prime}$.

For any round $r$ of $\mathfrak{R}$, we start by estimating an upper bound for $v_{i}(r)$ when $p_{i} \in \tilde{V}_{c}$. There are two cases. First, if $r \in \mathfrak{R}_{i}$, we have immediately $v_{i}(r) \leq v_{a}$. Second, when $r \notin \mathfrak{R}_{i}$, we have $v_{i}(r)>v_{a}$ but we know that after a finite number of rounds we will have again a round $r^{\prime}$ such that $r^{\prime} \in \mathfrak{R}_{i}$ (and so $v_{i}\left(r^{\prime}\right) \leq v_{a}$ ). There exists an upper bound $\theta$ on the difference $r^{\prime}-r \leq \theta<+\infty$ ( $\theta$ can be any large integer but not infinite). We compute now an over estimation of the maximum decrease of the value of $p_{i}$ (during $\theta$ consecutive rounds). In an extreme scenario, from round $r$ till round $r^{\prime}-1, p_{i}$ always uses its own value and $n-1$ values $v_{a}-\delta_{1}(r)$ when it computes an average (line 6). Consequently, when the round $r^{\prime}$ begins, we have $\frac{v_{i}(r)+\left(n^{\theta}-1\right)\left(v_{a}-\delta_{1}(r)\right)}{n^{\theta}} \leq v_{i}\left(r^{\prime}\right) \leq v_{a}$. From this formula, we extract an upper bound for $v_{i}(r)$ which is equal to $v_{a}+\left(n^{\theta}-1\right) \delta_{1}(r)$. Thus we have $v_{i}(r) \leq v_{a}$ if $r \in \mathfrak{R}_{i}$ and $v_{i}(r) \leq v_{a}+\left(n^{\theta}-1\right) \delta_{1}(r)$ if $r \notin \mathfrak{R}_{i}$. We select the greater value $v_{a}+\left(n^{\theta}-1\right) \delta_{1}(r)$ as the upper bound of $v_{i}(r)$. Note that $v_{a}+\left(n^{\theta}-1\right) \delta_{1}(r)$ is also the upper bound of $v_{i}(\tilde{r})$ for any round $\tilde{r}>r$. Again if $\tilde{r} \in \mathfrak{R}_{i}$, we have $v_{i}(\tilde{r}) \leq v_{a}$. If $\tilde{r} \notin \mathfrak{R}_{i}$, by using the same extreme estimation, we get $v_{i}(\tilde{r}) \leq v_{a}+\left(n^{\theta}-1\right) \delta_{1}(\tilde{r})$. As $\delta_{1}$ is non-increasing, we have $\delta_{1}(\tilde{r}) \leq \delta_{1}(r)$. Thus $v_{i}(\tilde{r}) \leq v_{a}+\left(n^{\theta}-1\right) \delta_{1}(r)$. Note that the validity property ensures that the lower bound of $v_{i}(r)$ is $v_{a}-\delta_{1}(r)$. Obviously, for any node $p_{i}$ of $\tilde{V}_{c}$, when $\delta_{1}(r)$ converges to 0 , the upper bound of $v_{i}(r)$ converges to $v_{a}$ and the lower bound of $v_{i}(r)$ converges also to $v_{a}$. Thus, $v_{i}(r)$ must converge to $v_{a}$. We conclude the existence of $V_{c}^{\prime}$ (which is equal to $\tilde{V}_{c}$ ).

Corollary 3. Suppose $\forall r \in \mathfrak{R}, \delta_{1}(r)>0$. For any correct node $p_{k}$, if $v_{k}$ does not converge to $v_{a}$, then $\exists r^{*} \in \mathfrak{R}$ and $\exists \ell_{\text {low }}>v_{a}$, such that $\forall r \geq r^{*}, v_{k}(r) \geq \ell_{\text {low }}$.

Proof: if $v_{k}$ does not converge to $v_{a}$, there exists a value $\alpha$ such that $\forall r \in \mathfrak{R}, \exists \hat{r}>r$, such that $v_{k}(\hat{r})-v_{a} \geq \alpha>0$. There are two cases corresponding to the non-convergence. First, since a round $r, v_{k}(r)$ is always greater than or equal to $v_{a}+\alpha$. In this case, $v_{a}+\alpha$ is a lower bound for $v_{k}$ since the round $r$. In the second case, from time to time $v_{k}$ is less than $v_{a}+\alpha$ and from time to time it is greater than or equal to $v_{a}+\alpha$. If this scenario happens, each time $v_{k}$ is less than $v_{a}+\alpha$, after a limited number of rounds, it must again become greater than or equal to $v_{a}+\alpha$. So $p_{k}$ must have infinite opportunities to compute an average with a value greater than $v_{a}+\alpha$. Formally, there is an infinite set $\mathfrak{R}_{k} \subset \mathfrak{R}$, such that $\forall r \in \mathfrak{R}_{k}$ node $p_{k}$ computes the average with at least a value greater than $v_{a}+\alpha$. For any consecutive elements $r, r^{\prime}$ of $\Re_{k}$, they satisfy $r^{\prime}-r \leq \theta<+\infty(\theta$ can be any large integer but not infinite). Consider a round $r \in \mathfrak{R}$ with $r>\theta$, we know $\exists \tilde{r} \in \mathfrak{R}_{k}$ with $r-\tilde{r} \leq \theta$. We consider an extreme scenario to estimate the lower bound of $v_{k}(r)$. Assume that $p_{k}$ computes an average with $v_{k}(\tilde{r}), v_{a}+\alpha$ and $(n-2)\left(v_{a}-\delta_{1}(\tilde{r})\right)$ during round $\tilde{r}$. Then from the round $\tilde{r}+1$ to the round $r-1, p_{k}$ continuously decrease its value by computing an average with its own value and $n-1$ values equal to $v_{a}-\delta_{1}(\tilde{r})$. Thus, when the round $r$ begins, we have $v_{k}(r) \geq \frac{v_{k}(\tilde{r})+\left(v_{a}+\alpha\right)+\left(n^{\theta}-1\right)\left(v_{a}-\delta_{1}(\tilde{r})\right)}{n^{\theta}}$. As $v_{k}(\tilde{r}) \geq v_{a}-\delta_{1}(\tilde{r})$, we get $v_{k}(r) \geq \frac{v_{a}+\alpha+n^{\theta}\left(v_{a}-\delta_{1}(\tilde{r})\right)}{n^{\theta}}$. As $\delta_{1}$ is non-increasing and converges to 0 , two properties hold: first $\frac{v_{a}+\alpha+n^{\theta}\left(v_{a}-\delta_{1}(\tilde{r})\right)}{n^{\theta}}$ is non-decreasing and second when $v_{a}+\alpha>n \delta_{1}(\tilde{r})$ we have $\frac{v_{a}+\alpha+n^{\theta}\left(v_{a}-\delta_{1}(\tilde{r})\right)}{n^{\theta}}>v_{a}$. Thus when the round $r$ is large enough, $v_{k}(r)$ has a lower bound $\ell_{\text {low }}>v_{a}$.

The twin corollary of Corollary 3 is given below.

Corollary 4. Suppose $\forall r \in \mathfrak{R}, \delta_{2}(r)>0$. For any correct node $p_{k}$, if $v_{k}$ does not converge to $v_{b}$, then $\exists r^{*} \in \mathfrak{R}$ and $\exists \ell_{u p}<v_{b}$, such that $\forall r \geq r^{*}, v_{k}(r) \leq \ell_{u p}$.

Note that Hypothesis 1 includes the following situation: $\exists r^{\prime} \in \mathfrak{R}$ such that $\forall r \geq r^{\prime}$, either $\delta_{1}(r)=0$ or $\delta_{2}(r)=0$. In the following, we simply write $\delta_{1}\left(r^{\prime}\right)=0$ or $\delta_{2}\left(r^{\prime}\right)=$ 0 when the value equal to 0 since a round $r^{\prime}$. We write $\delta_{1}(r)>0$ or $\delta_{2}(r)>0$ when the value is greater than 0 in any round $r$. There are three possible cases: (1) $\delta_{1}(r)>0$ and $\delta_{2}\left(r^{\prime}\right)=0 ;(2) \delta_{1}\left(r^{\prime}\right)=0$ and $\delta_{2}(r)>0 ;(3) \delta_{1}(r)>0$ and $\delta_{2}(r)>0$. In each case, we consider a partition of $V_{c}$ into three subsets. We use the same notation $\left\langle V_{c}^{1}, V_{c}^{2}, V_{c}^{3}\right\rangle$ to refer to this partition. The definitions of the sets are different in each case. If $\delta_{1}(r)>0$, then based on Lemma $4, V_{c}^{1}=$ $\left\{p_{i} \mid p_{i} \in V_{c} \wedge v_{i}\right.$ converges to $\left.v_{a}\right\}$. If $\delta_{1}\left(r^{\prime}\right)=0$, then $V_{c}^{1}=\left\{p_{i} \mid p_{i} \in V_{c} \wedge \forall r \geq r^{\prime}, v_{i}(r)=v_{a}\right\}$. Symmetrically, If $\delta_{2}(r)>0, V_{c}^{2}=\left\{p_{j} \mid p_{j} \in V_{c} \wedge v_{j}\right.$ converges to $\left.v_{b}\right\}$. If $\delta_{2}\left(r^{\prime}\right)=0, V_{c}^{2}=\left\{p_{j} \mid p_{j} \in V_{c} \wedge \forall r \geq r^{\prime}, v_{j}(r)=v_{b}\right\}$. Whatever the situation, $V_{c}^{3}=V_{c} /\left(V_{c}^{1} \cup V_{c}^{2}\right)$ : the third set contains the remaining correct nodes.

Lemma 5. Hypothesis 1 is false.

Proof: Clearly, $\left\langle F, L=V_{c}^{1}, M=V_{c}^{3}, R=V_{c}^{2}\right\rangle$ forms a $\digamma$ partition and belongs to the set $\Gamma$. The first conclusion is $\exists r^{*} \in \mathfrak{R}$, such that $\forall r \geq r^{*}$ both $\max (L, r)<\min (\mathbb{R}, r)$ and $\min (R, r)>\max (\mathbb{L}, r)$ are satisfied. Consider the set $L$. There are two cases. First, $\delta_{1}\left(r^{\prime}\right)=0$. The definition of $V_{c}^{1}$ in this case implies $\exists r^{*} \geq r^{\prime}$, such that $\forall r \geq r^{*}$ only the correct nodes in $L$ still hold the minimum value $v_{a}$. Consequently, $\forall r \geq r^{*}, \max (L, r)<\min (\mathbb{R}, r)$. Second, $\delta_{1}(r)>0$. In this case we know $\forall p_{i} \in L, v_{i}$ converges to $v_{a}$ (Lemma 4). Furthermore, $\forall p_{k} \in M \cup R, v_{k}$ does not converge to $v_{a}$ determines it has a lower bound $\ell_{\text {low }}>$ $v_{a}$ (Corollary 3). Therefore, $\exists r^{*} \in \mathfrak{R}$, such that $\forall r \geq r^{*}$, $\max (L, r)<\min (\mathbb{R}, r)$. By considering the set $R$, we can conclude $\exists r^{*} \in \mathfrak{R}$, then $\forall r \geq r^{*}, \min (R, r)>\max (\mathbb{L}, r)$. The proof is symmetric. 
The above conclusion ensures Lemma 2 can be applied in each round $r \geq r^{*}$. Although different values of $\delta_{1}$ and $\delta_{2}$ leads to three different cases, whatever the case, based on Lemma 2 and the fact that the number of nodes is finite, we can infer that at least one node on one side $\left(\exists p_{i} \in L\right.$ and/or $\exists p_{j} \in R$ ) has infinite opportunities to update its value with a value from $\left(v_{a}, v_{b}\right)$. Without loss of generality, suppose since a round $r^{*}, \exists p_{i} \in L$, such that $p_{i}$ has infinite opportunities to update its value with a value that has a lower bound greater than $v_{a}$. Formally, there exists an infinite set $\mathfrak{R}_{i} \subset \mathfrak{R}$, such that $\forall r \in \mathfrak{R}_{i}$ node $p_{i}$ computes the average with at least a value with a lower bound greater than $v_{a}$. The infinite property of $\mathfrak{R}_{i}$ ensures that for any consecutive elements $r, r^{\prime}$ of $\Re_{i}$, they satisfy $r^{\prime}-r \leq \theta<+\infty$ ( $\theta$ can be any large integer but not infinite). The way we have proved Corollary 3 also works here. By using an extreme estimation, we can conclude there exists a round and from then on $v_{i}$ has lower bound greater than $v_{a}$. This contradicts the fact that $p_{i} \in L$ ( $L=V_{c}^{1}$ ) which means that either $v_{i}$ converges to $v_{a}$ or is always equal to $v_{a}$. Hypothesis 1 is false.

Theorem 3. Approximate Byzantine consensus can be solved in $G$ if and only if $G$ is $f$-resilient.

\section{VI. $h$-DISJOINT AND $f$-RESILIENT}

As mentioned in Section IV, the use of the primitive 3-partial multicast has been investigated to solve exact Byzantine consensus. Based on uniform hyperedges, the property $h$-disjoint proposed in [4] is proved to be necessary and sufficient for reaching exact Byzantine consensus. The properties $h$-disjoint and $f$-resilient provide different (but strongly related) requirements on the communication topology. An interesting future work would be to determine if there exists a generalized property that copes with both exact Byzantine consensus and approximate Byzantine consensus. The comparison of $h$-disjoint and $f$-resilient done in this paper is a first step in this direction.

We summarize the property $h$-disjoint first. It requires that for all disjoint nodes subsets $A, B, C$, they satisfy the follow condition, $\exists p_{i}^{\prime} \in A, \exists p_{j}^{\prime} \in B, \exists p_{k}^{\prime} \in C$, such that $\left(p_{i}^{\prime}, p_{j}^{\prime}, p_{k}^{\prime}\right)$ is a uniform hyperedge and $|A|,|B|,|C| \geq h,|A|+|B|+$ $|C| \geq \frac{n+3 h}{2}$. For a uniform hypergraph $\bar{H}$ with $2 f+1 \leq$ $n \leq 3 f$, the exact Byzantine consensus is solvable if and only if $H$ is $(n-2 f)$-disjoint.

To do a comparison, a standard model is needed to cover both the uniform hypergraph $H$ and the hybrid graph $G$. We expand $H$ and define unicast channels: $H=\left(\mathcal{V}, \mathcal{E}_{U}, \mathcal{E}_{M}\right)$. There is a one-one mapping between the two sets $\mathcal{V}$ and $V$. We use the notation $p_{i}^{\prime} \asymp p_{i}\left(p_{i}^{\prime} \in \mathcal{V}, p_{i} \in V\right)$ to express it. Suppose $|\mathcal{V}|=|V|=n$ and $2 f+1 \leq n \leq 3 f$. Regarding the unicast channels, the dual sets $\mathcal{E}_{U}$ and $E_{U}$ are equivalent: $\left(p_{i}^{\prime} ; p_{j}^{\prime}\right) \in \mathcal{E}_{U}$ if and only if $\left(p_{i} ; p_{j}\right) \in E_{U}$. According to $h$-disjoint, the set $\mathcal{E}_{M}$ only contains uniform hyperedges. We assume that $\left(p_{i}^{\prime}, p_{j}^{\prime}, p_{k}^{\prime}\right) \in \mathcal{E}_{M}$ if and only if $\left(p_{i} ; p_{j}, p_{k}\right),\left(p_{j} ; p_{i}, p_{k}\right),\left(p_{k} ; p_{i}, p_{j}\right) \in E_{M}$. The connectivity of both communication graphs $H$ and $G$ is at least $2 f+1$, which implies that each node has at least $2 f+1$ source neighbors (an exception is $n=2 f+1$, if so, consider each node has $2 f$ source neighbors).

Lemma 6. Let $n=2 f+h(1 \leq h \leq f)$. If $H$ is not $h$-disjoint, then $G$ is not $f$-resilient.

Proof: When $H$ is not $h$-disjoint, there exists three subsets $A, B, C \subseteq \mathcal{V}$ such that $|A|,|B|,|C| \geq h,|A|+$ $|B|+|C| \geq(n+3 h) / 2$, and $\forall p_{i}^{\prime} \in A, \forall p_{j}^{\prime} \in B, \forall p_{k}^{\prime} \in C$, $\left(p_{i}^{\prime}, p_{j}^{\prime}, p_{k}^{\prime}\right) \notin \mathcal{E}_{3}$. Due to $|A|,|B|,|C| \geq h$, we can conclude that at most one set among $A, B$ and $C$ can have a cardinality greater than $f$ (otherwise $|A|+|B|+|C|>n$ ). The proof below includes two cases. First, among $A, B, C$ only one set has a cardinality greater than $f$ and second, all the three sets have a cardinality less than or equal to $f$. Due to $|A|+|B|+|C|$ may be less than $n$, suppose $D=\mathcal{V} /(A \cup B \cup C$ ) (if $A \cup B \cup C=\mathcal{V}, D=\emptyset$ ).

Consider the first case: among $A, B, C$ only one set has a cardinality greater than $f$. Without loss of generality, suppose $|A|>f$. Let $A^{\prime} \subset A$ and $|A|-\left|A^{\prime}\right|=f$. Due to $|A|>f$, we know $A^{\prime} \neq \emptyset$. Consider the $\digamma$ partition of $V$ with $F \asymp A / A^{\prime}, L \asymp B, M \asymp D \cup A^{\prime}$ and $R \asymp C$. Note that no uniform hyperedges among $A, B, C$ implies no 3-partial multicast channel among $F, L$ and $R$. Due to $|F|=f,|L|=$ $|B| \geq h,|R|=|C| \geq h$ and $n=2 f+h$, we have $\forall p_{i} \in L$, $\forall p_{j} \in R,\left|N_{i} \cap \mathbb{R}\right| \leq|\mathbb{R}| \leq f$ and $\left|N_{j} \cap \mathbb{L}\right| \leq|\mathbb{L}| \leq f$. Consequently, $\left|F_{i j}\right|+\left|N_{i} \cap \mathbb{R}\right|+\left|N_{j} \cap \mathbb{L}\right| \leq 0+f+f<2 f+1$, $G$ is not $f$-resilient.

Now consider the second case: among $A, B, C$ no set has a cardinality greater than $f$. There are two subcases depending on $|A|+|D| \leq f$ or $|A|+|D|>f$. Consider $|A|+|D| \leq f$ first. Let $B^{\prime} \subset B, C^{\prime} \subset C$ with $|A|+\left|B^{\prime}\right|+$ $\left|C^{\prime}\right|+|D|=f$ (if $|A|+|D|=f$, then $B^{\prime}=C^{\prime}=\emptyset$ ). Consider the following $\digamma$ partition of $V$ with $F \asymp A \cup B^{\prime} \cup$ $C^{\prime} \cup D, L \asymp B / B^{\prime}, M \asymp \emptyset$ and $R \asymp C / C^{\prime}$. Note that $|A| \geq h$ and no uniform hyperedge is among $A, B, C$, such that $\forall p_{i} \in L, \forall p_{j} \in R$, we have $\left|F_{i j}\right| \leq f-h$. Moreover, $M=\emptyset$ implies $|\mathbb{L}|+|\mathbb{R}|=2 f+h-f=f+h$. Consequently, $\left|F_{i j}\right|+\left|N_{i} \cap \mathbb{R}\right|+\left|N_{j} \cap \mathbb{L}\right| \leq f-h+f+h=2 f<2 f+1, G$ is not $f$-resilient in this subcase. Now consider $|A|+|D|>f$. To facilitate the presentation, we include four parameters $a, b, c, d$ to express $|A|,|B|,|C| \geq h:|A|=h+a,|B|=$ $h+b,|C|=h+c$ and $|D|=d$. Due to $|A|+|B|+|C| \geq$ $(n+3 h) / 2=f+2 h$, thus $d \leq n-(f+2 h)=f-h .|A|<f$ and $|A|+|D|>f$ in this case implies $\exists D^{\prime} \subset D$, such that $\left|D^{\prime}\right|+|A|=f$. Let $\left|D^{\prime}\right|=d^{\prime}$, thus $d^{\prime}+a=f-h$. Note that we also have $a+b+c+d=2 f+h-3 h=2 f-2 h$, such that $b+c=2 f-2 h-\left(d^{\prime}+a+d-d^{\prime}\right)$. Due to $d^{\prime}+a=f-h$, thus $b+c=f-h-\left(d-d^{\prime}\right)$. Consider the $\digamma$ partition of $V$ with $F \asymp A \cup D^{\prime}, L \asymp B, M \asymp D / D^{\prime}$ and $R \asymp C$, such that $|\mathbb{L}|=h+b+d-d^{\prime}$ and $|\mathbb{R}|=h+c+d-d^{\prime}$. No uniform hyperedge is among $A, B, C$, thus $\forall p_{i} \in L$ 
and $\forall p_{j} \in R$, we have $\left|F_{i j}\right| \leq\left|D^{\prime}\right|=d^{\prime}$. Consequently, $\left|F_{i j}\right|+\left|N_{i} \cap \mathbb{R}\right|+\left|N_{j} \cap \mathbb{L}\right| \leq\left|F_{i j}\right|+|\mathbb{R}|+|\mathbb{L}| \leq d^{\prime}+(h+$ $\left.b+d-d^{\prime}\right)+\left(h+c+d-d^{\prime}\right)=2 h+2 d-d^{\prime}+(b+c)$. We already get $d \leq f-h$ and $b+c=f-h-\left(d-d^{\prime}\right)$, thus $2 h+2 d-d^{\prime}+(b+c)=h+f+d \leq h+f+f-h<2 f+1$, $G$ is not $f$-resilient.

In another aspect, $H$ is $h$-disjoint does not always mean $G$ is $f$-resilient. We find a counterexample in [6] (page 452). A 1-disjoint hypergraph for $n=5$ and $f=2$ is provided, but this example cannot satisfy 2 -resilient.

\section{ASYNCHRONOUS NETWORKS}

In asynchronous networks, the message transmission and processing delay is arbitrary but finite. Under the model asynchrony, no correct node can differentiate if another node is just slow or stops working. Therefore, in each algorithm round, a correct node $p_{i}$ can only expect to receive the values from its $\left|N_{i}\right|-f$ sources neighbors. Solving approximate Byzantine consensus in asynchronous networks also benefits from the 3-partial multicast primitive. We claim that the idea behind $f$-resilient can be extended straightforward into asynchronous networks by increasing the requirement of cardinalities (see Definition 6).

Definition 6. $\langle F, L, M, R\rangle$ is an asy-safe $\digamma$ partition of $G$ if and only if one of the following properties holds:

(1) $\exists p_{i} \in L$ such that $\left|N_{i} \cap \mathbb{R}\right| \geq 2 f+1$ or $\exists p_{j} \in R$ such that or $\left|N_{j} \cap \mathbb{L}\right| \geq 2 f+1$;

(2) $\exists p_{i} \in L$ and $\exists p_{j} \in R$, such that $f+1 \leq\left|N_{i} \cap \mathbb{R}\right| \leq 2 f$, $f+1 \leq\left|N_{j} \cap \mathbb{L}\right| \leq 2 f,\left|F_{i j}\right|+\left|N_{i} \cap \mathbb{R}\right|+\left|N_{j} \cap \mathbb{L}\right| \geq 4 f+1$.

\section{CONCLUSION}

We propose a novel condition $f$-resilient, which takes into account both unicast and partial multicast communication primitives. $f$-resilient is proved to be necessary and sufficient for reaching iterative approximate consensus in synchronous networks. The relationships between $h$-disjoint and $f$-resilient are also investigated. Finally, the idea behind $f$-resilient is extended to cope with asynchronous networks.

\section{ACKNOWLEDGMENT}

This work is partially supported by National $973 \mathrm{Hi}-\mathrm{Tech}$ Program, China \& by a Mayenne's military project NoPEC.

\section{REFERENCES}

[1] D. Dolev, A. N. Lynch, S. Pinter, W. E. Stark, and E. W. Weihl. Reaching approximate agreement in the presence of faults. In proc. of the IEEE symp. on reliability in distributed software and database systems, pages 145-154, 1983.

[2] N. Vaidya, L. Tseng, and G. Liang. Iterative approximate byzantine consensus in arbitrary directed graphs. In proc. of the ACM symp. on principles of distributed computing, pages 365-374, 2012.
[3] H. LeBlanc, H. Zhang, S. Sundaram, and X. Koutsoukos. Consensus of multi-agent networks in the presence of adversaries using only local information. In proc. of the Int. conf. on high confidence networked systems, pages 1-10, 2012.

[4] A. Jaffe, T. Moscibroda, and S. Sen. On the price of equivocation in byzantine agreement. In proc. of the ACM symp. on principles of distributed computing, pages 309-318, 2012.

[5] M. Fitzi and U. Maurer. From partial consistency to global broadcast. In proc. of the ACM symp. on theory of computing, pages 494-503, 2000.

[6] D.V.S. Ravikant, V. Muthuramakrishnan, V. Srikanth, K. Srinathan, and C. P. Rangan. On byzantine agreement over $(2,3)-$ uniform hypergraphs. In proc. of the Int. symp. on distributed computing, pages 450-464, 2005.

[7] M. Franklin and M. Yung. Secure hypergraphs: Privacy from partial broadcast. In proc. of the ACM symp. on the theory of computing, pages 36-44, 1995.

[8] M. H. Azadmanesh and H. Bajwa. Global convergence in partially fully connected networks (PFCN) with limited relays. In proc. of the IEEE conf. on industrial electronics society, pages 2022-2025, 2001.

[9] M. H. Azadmanesh and A. W. Krings. A step toward global convergence in partially connected networks. In proc. of the Int. conf. on parallel and distributed computing systems, pages 234-241, 1997.

[10] S. Sundaram and C. N. Hadjicostis. Distributed function calculation via linear iterations in presence of malicious agents - part i: Attacking the networks. In proc. of the American control conf., pages 1350-1355, 2008.

[11] S. Sundaram and C. N. Hadjicostis. Distributed function calculation via linear iterations in presence of malicious agents - part ii: Overcoming malicious behavior. In proc. of the American control conf., pages 1356-1361, 2008.

[12] C. Li, M. Hurfin, and Y. Wang. Approximate byzantine consensus in sparse, mobile ad-hoc networks. Journal of Parallel and Distributed Computing, Elsevier, 74:2860-2871, June 2014.

[13] B. Charron-Bost, M. Függer, and T. Nowak. Approximate consensus in highly dynamic networks: The role of averaging algorithms. In proc. of the Int. Conf. on Automata, Languages, and Programming - ICALP, pages 528-539, 2015.

[14] I. Abraham, Y. Amit, and D. Dolev. Optimal resilience asynchronous approximate agreement. In proc. of the Int. conf. on principles of distributed Systems, LNCS 3544, pages 229-239, 2005.

[15] A. Clement, F. Junqueira, A. kate, and R. Rodrigues. On the (limited) power of non-equivocation. In proc. of the ACM symp. on principles of distributed computing, pages 301-308, 2012.

[16] M. Backes, F. Bendun, A. Choudhury, and A. Kate. Asynchronous MPC with a strict honest majority using nonequivocation. In proc. of the ACM symp. on principles of distributed computing, pages 10-19, 2014. 